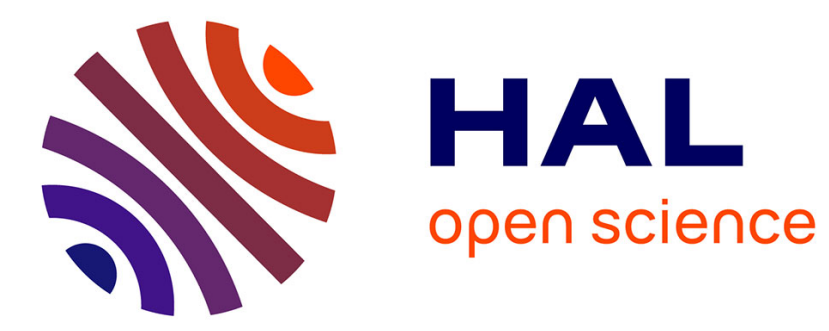

\title{
Attachement électronique dissociatif sur HCI et DCI
}

\author{
R. Azria, L. Roussier, R. Paineau, M. Tronc
}

\section{To cite this version:}

R. Azria, L. Roussier, R. Paineau, M. Tronc. Attachement électronique dissociatif sur HCI et DCI. Revue de Physique Appliquée, 1974, 9 (2), pp.469-473. 10.1051/rphysap:0197400902046900 . jpa00243804

\section{HAL Id: jpa-00243804 https://hal.science/jpa-00243804}

Submitted on 1 Jan 1974

HAL is a multi-disciplinary open access archive for the deposit and dissemination of scientific research documents, whether they are published or not. The documents may come from teaching and research institutions in France or abroad, or from public or private research centers.
L'archive ouverte pluridisciplinaire HAL, est destinée au dépôt et à la diffusion de documents scientifiques de niveau recherche, publiés ou non, émanant des établissements d'enseignement et de recherche français ou étrangers, des laboratoires publics ou privés. 


\title{
ATTACHEMENT ÉLECTRONIQUE DISSOCIATIF SUR HCI ET DCI
}

\author{
R. AZRIA, L. ROUSSIER, R. PAINEAU et M. TRONC
}

Laboratoire de Collisions Electroniques (*)

Université Paris-Sud, 91405 Orsay, France

(Reçu le 5 juin, révisé le 10 octobre 1973)

\begin{abstract}
pics à 6,9 et 9,2 eV. Ces pics sont attribués aux ions $\mathrm{H}^{-}\left(\mathrm{D}^{-}\right)$. mesuré les effets isotopiques correspondants. Nous obtenons :

$\begin{array}{rll}\text { à } 0,84 \mathrm{eV} & \sigma_{(\mathrm{Cl}-/ \mathrm{HCl})}=(8,9 \pm 0,7) 10^{-18} \mathrm{~cm}^{2} \\ \text { et } & \sigma_{(\mathrm{Cl}-/ \mathrm{HCl})} / \sigma_{(\mathrm{Cl}-/ \mathrm{DCl})}=5 \pm 1,1 ; \\ \text { à } 6,9 \mathrm{eV} & \sigma_{(\mathrm{H}-/ \mathrm{HCl})}=(5,2 \pm 0,4) 10^{-19} \mathrm{~cm}^{2} \\ & \text { et } & \sigma_{(\mathrm{H}-/ \mathrm{HCl})} / \sigma_{(\mathrm{D}-/ \mathrm{DCl})}=1,8 \pm 0,1 ; \\ \text { à } 9,2 \mathrm{eV} & \sigma_{(\mathrm{H}-/ \mathrm{HCl})}=(2,8 \pm 0,2) 10^{-19} \mathrm{~cm}^{2} \\ & \text { et } & \sigma_{(\mathrm{H}-/ \mathrm{HCl})} / \sigma_{(\mathrm{D}-/ \mathrm{DCl})} \geqslant 1,4 .\end{array}$
\end{abstract}

Résumé. - La formation des ions négatifs dans $\mathrm{HCl}$ et $\mathrm{DCl}$ est étudiée à l'aide d'un tube à ionisation totale. En plus du pic d'ions $\mathrm{Cl}^{-}$observé par de nombreux auteurs, la courbe représentant le courant d'ions négatifs en fonction de l'énergie des électrons comporte deux autres

Nous avons déterminé la valeur des sections efficaces des différents processus d'attachement et

Abstract. - The formation of negative ions in $\mathrm{HCl}$ and $\mathrm{DCl}$ is studied with a total ionization chamber. In addition to the already well established peak of $\mathrm{Cl}^{-}$ions, two other peaks are observed at 6.9 and $9.2 \mathrm{eV}$. These peaks are due to $\mathrm{H}^{-}\left(\mathrm{D}^{-}\right)$ions.

We have determined the absolute cross section for the different processes and measured the corresponding isotope effects. We obtained :

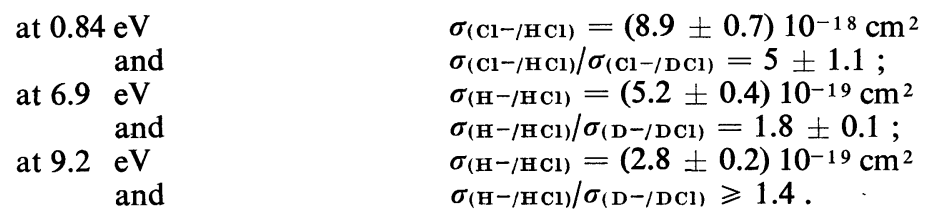

1. Introduction. - L'attachement électronique dissociatif sur $\mathrm{HCl}$ a été étudié par de nombreux auteurs, utilisant des méthodes variées : spectrométrie de masse [1]-[6], ionisation totale [7], et enfin une méthode combinant la spectrométrie de masse avec des expériences à une pression de quelques torrs « swarmbeam method») [8]. Dans ces études, seul un pic d'ions $\mathrm{Cl}^{-}$, à environ $0,7 \mathrm{eV}$, est observé en fonction de l'énergie des électrons.

Les sections efficaces de formation des ions $\mathrm{Cl}^{-}$ dans $\mathrm{HCl}$ ont été mesurées par R. N. Compton [8] d'une part et I. S. Buchel'Nikova [7] d'autre part.

R. N. Compton et ses collaborateurs ont de plus mesuré la section efficace de formation des ions $\mathrm{Cl}^{-}$ dans $\mathrm{DCl}$, et obtiennent la valeur de 1,4 pour le rapport

$$
\sigma_{(\mathrm{Cl}-/ \mathrm{HCl})} / \sigma_{(\mathrm{Cl}-/ \mathrm{DCl})},
$$

l'azote étant utilisé comme gaz porteur.

(*) Associé au CNRS.
Comme nous allons le voir, cette valeur est en désaccord avec celle que nous obtenons.

Notre étude a de plus permis de mettre en évidence deux autres états de l'ion négatif $\mathrm{HCl}^{-}$, corrélés aux fragments $\mathrm{H}^{-}$et $\mathrm{Cl}$.

2. Appareillage. Méthode de mesure. - L'appareil à ionisation totale utilisé est formé d'un canon à électrons et d'une chambre de collisions à plaques parallèles. Ces éléments ont été décrits en détail lors de précédentes études [9], [10]. La figure 1 donne un schéma d'ensemble de l'appareil :

- Le canon à électrons permet l'utilisation de la méthode de la différence du potentiel retardateur (RPD) [11] afin de réduire la composante longitudinale de la distribution énergétique des électrons.

- Les ions sont collectés sur la plaque $P_{1}$. Pour être sûr de collecter tous les ions formés, on augmente le champ électrique entre les plaques $P_{1}$ et $P_{2}$, jusqu'au moment où la courbe représentant le courant d'ions en fonction du champ électrique atteint un palier 


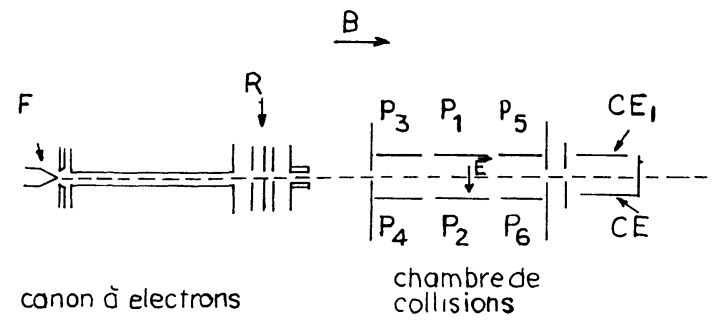

FIG. 1. - Schéma du tube à ionisation totale. (Le rôle des différentes plaques est défini dans la référence [10].)

(Fig. 2). Ce palier est atteint rapidement dans le cas des ions $\mathrm{Cl}^{-}$car leur énergie cinétique est très faible, mais il est atteint lentement dans le cas des ions $\mathrm{H}^{-}$ car leur énergie cinétique est grande. Remarquons que l'intensité des ions $\mathrm{Cl}^{-}$décroît quand le champ extracteur est augmenté au-delà de $2 \mathrm{~V} / \mathrm{cm}$; nous pensons que la distribution en énergie du faisceau d'électrons incidents s'élargit en présence d'un champ extracteur élevé, ce qui a pour effet de diminuer le courant d'ions quand le pic d'attachement résonnant est très étroit ; on peut voir sur la figure 3 que le pic d'attachement est beaucoup plus étroit pour $\mathrm{Cl}^{-}$ que pour $\mathrm{H}^{-}$, donc beaucoup plus sensible à la résolution du faisceau d'électrons incidents.

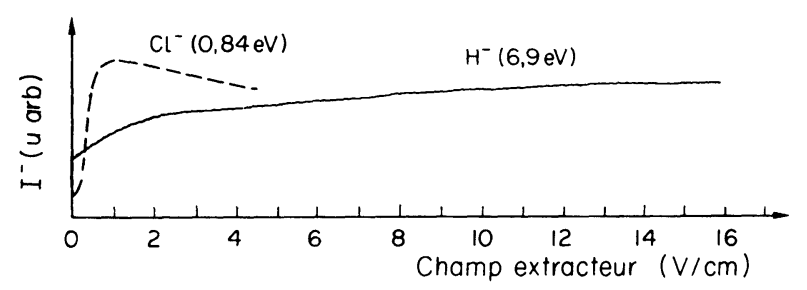

Fig. 2. - Variation du courant d'ions négatifs collectés, en fonction du champ extracteur. Les chiffres entre parenthèses indiquent l'énergie moyenne des électrons incidents.

Si on inverse la direction du champ électrique, les ions doivent surmonter une barrière de potentiel pour être collectés sur la plaque $P_{1}$. Pour une énergie des électrons donnée, le potentiel retardateur maximum que les ions peuvent franchir est une mesure de l'énergie cinétique maximum de ces ions.

Pour mesurer la section efficace de formation des ions négatifs, on mesure pour chaque pic le rapport entre le courant total d'ions négatifs $I^{-}$et le courant total d'ions positifs $I^{+}$, à $75 \mathrm{eV}$. La valeur absolue des sections efficaces est alors obtenue en multipliant ces rapports par la valeur absolue de la section efficace d'ionisation positive totale à $75 \mathrm{eV}$.

$\mathrm{HCl}$ a été fourni par Mathesson $\mathrm{C}^{\text {ie }}$; la pureté est de $99,99 \%$.

$\mathrm{DCl}$ a été fourni par Merk, Sharp \& Dhome; la pureté à la mise en bouteille est de $99 \%$.

Les expériences sur $\mathrm{HCl}$ et $\mathrm{DCl}$ ont été faites avec différentes valeurs du courant électronique $(10$ à $100 \mathrm{nA}$ ), les pressions de gaz utilisées variant de $10^{-5}$ à $5 \times 10^{-4}$ torr.

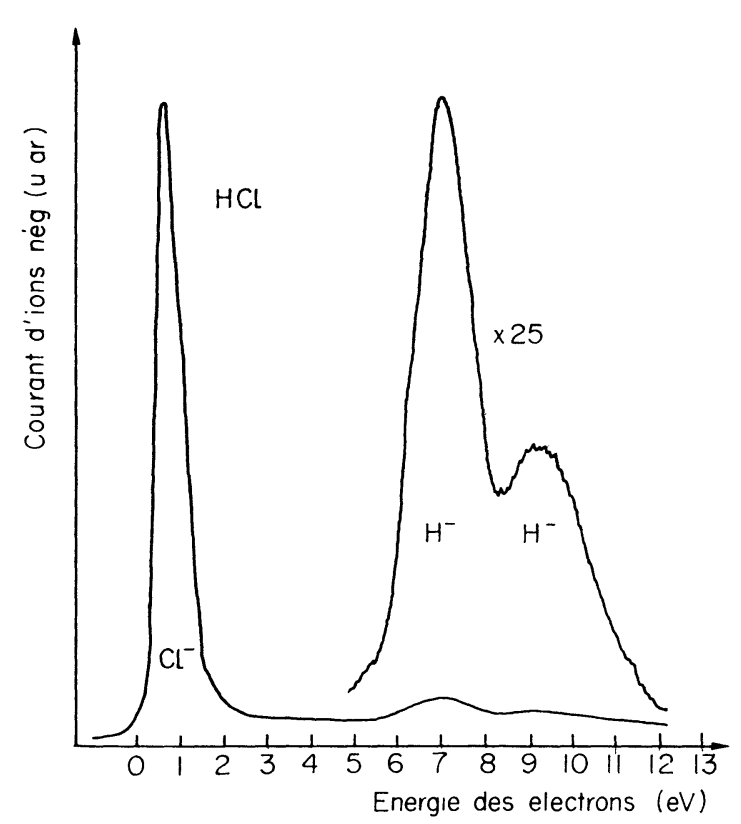

FIG. 3. - Formation d'ions négatifs par attachement dissociatif dans $\mathrm{HCl}$.

La très grande adsorption de ces gaz sur les parois de l'enceinte et sur les différentes plaques constituant le canon à électrons et la chambre de collisions, a rendu ces expériences très délicates. Cette adsorption se manifestait essentiellement par :

- une baisse continue de la pression dans la chambre de collisions ;

- des variations de l'échelle d'énergie au cours du temps, dues à la modification des potentiels de contact entre les différentes plaques ;

- une détérioration très rapide des résistances d'isolement entre les plaques de la chambre de collisions.

3. Résultats. - 3.1 SPeCtRe DeS IONS NÉGATIFS FORMÉS PAR ATTACHEMENT DISSOCIATIF SUR $\mathrm{HCl}$. COURBE D'IONISATION DE $\mathrm{Cl}^{-}$. - La figure 3 représente le courant d'ions négatifs dans $\mathrm{HCl}$ en fonction de l'énergie des électrons incidents. Cette courbe, obtenue sans utiliser la méthode « RPD », présente 3 pics :

- le $1^{\text {er }}$ (seuil à $0,74 \mathrm{eV}$ et maximum à $0,84 \mathrm{eV}$ ) est dû aux ions $\mathrm{Cl}^{-}$;

- les $2^{\mathrm{e}}$ et $3^{\mathrm{e}}$ pics (maximum à 6,9 et $9,2 \mathrm{eV}$ ) sont dus aux ions $\mathrm{H}^{-}$. L'identification de ces ions a été faite en spectrométrie de masse au laboratoire.

A cause de la variation des potentiels de contact signalée plus haut, il ne nous a pas été possible d'obtenir un étalonnage à partir de la courbe d'établissement du courant électronique. L'échelle d'énergie proposée a été obtenue par J. P. Ziesel $\left(^{*}\right)$ [12] à partir du pic d'ions $\mathrm{O}^{-} / \mathrm{CO}$, puisque le potentiel d'apparition

(*) Détaché à Mason Laboratory, Yale University, New Haven, Connecticut 06520, U.S. A. 
comme la position du maximum de la section efficace sont connus avec précision pour ce processus [13].

R. N. Compton et ses collaborateurs [8] ont été les premiers à observer un maximum secondaire à côté du maximum principal, à $0,78 \mathrm{eV}$, dans la courbe d'ionisation de $\mathrm{Cl}^{-}$. Nous avons cherché, sans succès, à reproduire cette structure en utilisant la méthode RPD. Cependant J. P. Ziesel [12] à l'aide d'un spectromètre de masse quadrupolaire utilisant un monochromateur trochoïdal d'électrons, a observé une série de maxima secondaires. Sur la figure 4, nous avons représenté notre courbe et celle de Ziesel, normalisées à la même hauteur du pic à $0,84 \mathrm{eV}$.

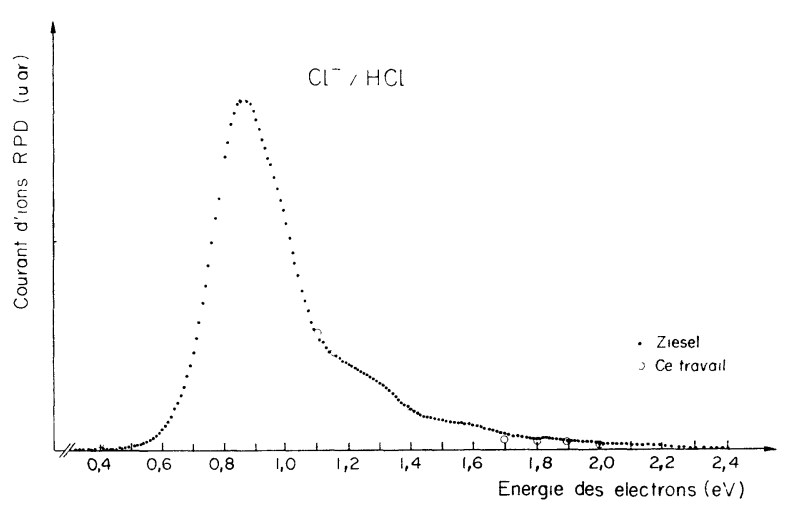

Fig. 4. - Forme du pic $\mathrm{Cl}^{-} / \mathrm{HCl}$. Comparaison entre nos mesures et celles de Ziesel et Schulz [12].

Une telle structure n'a pas été observée dans la courbe d'ionisation de $\mathrm{Cl}^{-} / \mathrm{DCl}$.

Les calculs de F. Fiquet-Fayard [14] montrent que cette structure peut être expliquée par l'ouverture de nouveaux canaux de sortie vibrationnels, $v$, pour l'état résonnant $\mathrm{HCl}^{-}$:

$$
\mathrm{HCl}^{-} \rightarrow \mathrm{e}+\mathrm{HCl}_{\mathrm{v}} \text {. }
$$

Nous voyons de plus sur la figure 4 , que la courbe de section efficace croît très vite au-dessus du seuil, ce qui indique qu'il s'agit d'un processus à seuil vertical.
3.2 MeSURE DES SECTIONS EFFICACES DE FORMATION DES IONS NÉGATIFS DANS HCl. EFFETS ISOTOPIQUES. 3.2.1 Sections efficaces dans $\mathrm{HCl}$. - Nous avons mesuré les sections efficaces de formation des ions négatifs dans $\mathrm{HCl}$ selon la méthode décrite au paragraphe 2. Les valeurs des rapports $I^{-} / I_{75 \mathrm{eV}}^{+}$sont reportées dans le tableau I. Ces rapports sont constants et indépendants de la pression comme on peut le voir sur les courbes de la figure 5 ; ces courbes représentent le courant d'ions à $0,84,6,9$ et $9,2 \mathrm{eV}$ en fonction du courant d'ions positifs $I^{+}$à $75 \mathrm{eV}$ pour diverses valeurs de la pression, le courant électronique étant de $1 \mathrm{nA}$.

Les valeurs absolues des sections efficaces (Tableau I) sont obtenues en multipliant les différents rapports $I^{-} / I^{+}$par la valeur de la section efficace d'ionisation positive totale dans $\mathrm{HCl}$ à $75 \mathrm{eV}\left(\sigma_{75 \mathrm{eV}}^{+}\right)$. Compton et Van Voorhis [15] ont mesuré $\sigma_{75 \mathrm{ev}}^{+}(\mathrm{HCl})$ : ils obtiennent $\sigma_{75 \mathrm{eV}}^{+}(\mathrm{HCl})=4,40 \times 10^{-16} \mathrm{~cm}^{2}$. Otvos et Ste-

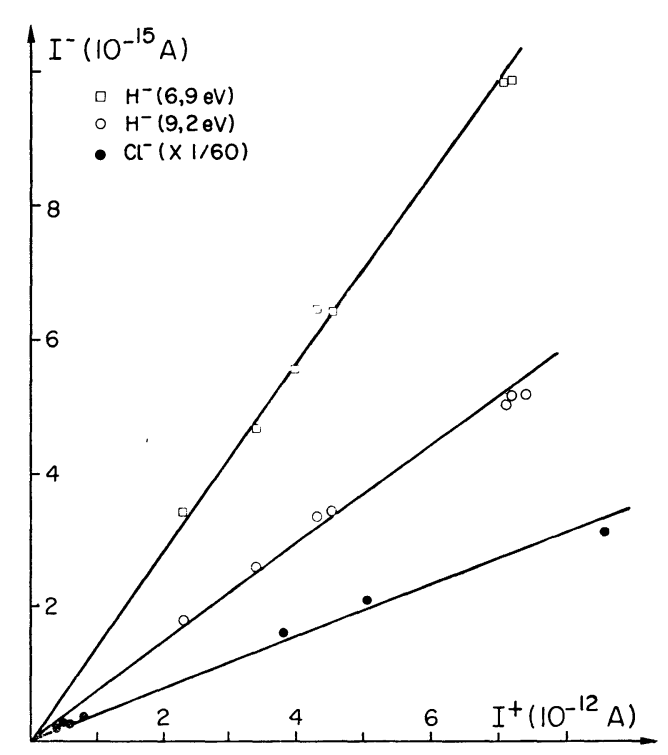

FIG. 5. - Courant d'ions négatifs en fonction du courant d'ions positifs à $75 \mathrm{eV}$.

\section{TABleAU I}

Sections efficaces d'attachement dans $\mathrm{HCl}$ et $\mathrm{DCl}$.

(Les erreurs quotées sur les sections efficaces sont des erreurs expérimentales sur la détermination de $\mathrm{I}^{-} / \mathrm{I}^{+}$).

\begin{tabular}{llccr}
$E_{\mathrm{ev}}$ & - & $\mathrm{HCl}$ & $\mathrm{DCl}$ & \multicolumn{1}{c}{$\sigma_{(\mathrm{HCl})} / \sigma_{(\mathrm{DCl})}$} \\
0,84 & $I^{-} / I_{75 \mathrm{ev}}^{+}$ & $(2,4 \pm 0,2) \times 10^{-2}$ & $0,5 \times 10^{-2}$ & $5 \pm 1,1$ \\
& $\sigma^{-} \mathrm{cm}^{2}$ & $(8,9 \pm 0,7) \times 10^{-18}$ & $1,8 \times 10^{-18}$ & \\
6,9 & $I^{-} / I_{75 \mathrm{eV}}^{+}$ & $(1,4 \pm 0,1) \times 10^{-3}$ & $0,77 \times 10^{-3}$ & $1,8 \pm 0,1$ \\
& $\sigma^{-} \mathrm{cm}^{2}$ & $(5,2 \pm 0,4) \times 10^{-19}$ & $2,9 \times 10^{-19}$ & \\
9,2 & $I^{-} / I_{75}^{+} \mathrm{ev}$ & $(0,74 \pm 0,06) \times 10^{-3}$ & $\leqslant 0,56 \times 10^{-3}$ & $\geqslant 1,4$ \\
& $\sigma^{-} \mathrm{cm}^{2}$ & $(2,8 \pm 0,2) \times 10^{-19}$ & $\leqslant 2,1 \times 10^{-19}$ &
\end{tabular}


venson [16], Lampe, Franklin et Field [17] ont mesuré $\sigma_{75 \mathrm{ev}}^{+}(\mathrm{HCl})$ relative à celle de l'argon. Ces valeurs sont respectivement 1,04 et 1,33 . Cette dernière a été retenue pour notre calcul. La valeur absolue de la section efficace d'ionisation dans l'argon mesurée par Rapp et Englander-Golden [18] $\left(2,8 \times 10^{-16} \mathrm{~cm}^{2}\right)$ est alors utilisée pour obtenir

$$
\sigma_{75 \mathrm{ev}}^{+}(\mathrm{HCl})=3,75 \times 10^{-16} \mathrm{~cm}^{2}
$$

Les sections efficaces de formation de $\mathrm{H}^{-}$n'ont pas été mesurées par d'autres auteurs, mais la section efficace de formation de $\mathrm{Cl}^{-}$a été mesurée antérieurement par Buchel'Nikova [7], qui trouve une valeur deux fois plus faible que la nôtre, et par Compton [8], qui trouve une valeur deux fois plus grande que la nôtre. Ces désaccords ne sont pas surprenants car la détermination des sections efficaces absolues est difficile. Par contre, l'erreur devrait être plus petite en ce qui concerne les sections efficaces relatives, et en particulier l'effet isotopique ; cependant nous allons voir maintenant qu'il existe un désaccord considérable entre la valeur que nous avons mesurée pour l'effet isotopique, et la valeur mesurée par Compton [8].

3.2.2 Effets isotopiques. - Les effets isotopiques sont généralement déterminés à l'aide d'un spectromètre de masse suivant la méthode décrite dans les références [9] et [19].

Pour les ions $\mathrm{Cl}^{-} / \mathrm{DCl}$ à $0,84 \mathrm{eV}$ et $\mathrm{D}^{-} / \mathrm{DCl}$ à .9,2 eV, nous avons cependant utilisé l'appareil à ionisation totale. En effet, d'une part notre spectromètre de masse ne permet pas d'atteindre des énergies inférieures à $1 \mathrm{eV}$, d'autre part l'énergie cinétique considérable des ions $\mathrm{D}^{-}$à $9,2 \mathrm{eV}(5,5 \mathrm{eV})$ entraînait une discrimination importante.

A $6,9 \mathrm{eV}$ nous obtenons au spectromètre de masse un effet isotopique

$$
\sigma_{\left(\mathrm{H}^{-} / \mathrm{HCl}^{\prime}\right)} / \sigma_{\left(\mathrm{D}^{-} / \mathrm{DCl}\right)}=1,8 \pm 0,1 .
$$

A 9,2 et $0,84 \mathrm{eV}$ nous obtenons à l'aide du tube à ionisation totale

$$
\sigma_{\left(\mathrm{D}^{-} / \mathrm{DCl}\right)}=2,1 \times 10^{-19} \mathrm{~cm}^{2}
$$

et

$$
\left.\sigma_{(\mathrm{Cl}-/ \mathrm{DCl}}\right)=2,3 \times 10^{-18} \mathrm{~cm}^{2},
$$

donc un effet isotopique de 3,9 en désaccord avec la valeur obtenue par Compton [1], [4]. Le désaccord est encore plus marqué si on tient compte des impuretés contenues dans notre ballon de $\mathrm{DCl}$; pour cela nous avons déterminé sa composition au spectromètre de masse. Nous avons obtenu :

$$
\begin{aligned}
(\mathrm{DCl})=86 \% ; & (\mathrm{HCl})=12 \% ; \\
\left(\mathrm{H}_{2} \mathrm{O}\right)=0,6 \% ; & \left(\mathrm{D}_{2} \mathrm{O}\right)=1,3 \% .
\end{aligned}
$$

A partir de ces concentrations, nous obtenons une section efficace de formation des ions $\mathrm{Cl}^{-}$dans $\mathrm{DCl}$ égale à $1,45 \times 10^{-18} \mathrm{~cm}^{2}$ donc un effet isotopique de 6,1 .

A cause des échanges isotopiques, la.composition du ballon évolue vers l'appauvrissement en $\mathrm{DCl}$ : donc 6,1 est une limite supérieure de l'effet isotopique. Nous pensons qu'au cours de nos expériences la teneur en $\mathrm{HCl}$ était inférieure à $12 \%$ et nous retenons pour l'effet isotopique la valeur $5 \pm 1,1$, moyenne entre 3,9 et 6,1 . Les différents résultats sont reportés dans le tableau I.

3.3 ENERGIE CINÉTIQUE DES IONS $\mathrm{H}^{-}$. - L'énergie cinétique maximum des ions $\mathrm{H}^{-}$à 6,9 et $9,2 \mathrm{eV}$ a été déterminée à partir des courbes représentant la variation du courant d'ions en fonction du champ retardateur entre les plaques $\mathbf{P}_{1}$ et $\mathbf{P}_{2}$. Nous mesurons ainsi une énergie cinétique de $3,3 \mathrm{eV}$ pour les ions $\mathrm{H}^{-}$à $6,9 \mathrm{eV}$ et $5,5 \mathrm{eV}$ pour les ions $\mathrm{H}^{-}$à $9,2 \mathrm{eV}$. Ces ions sont donc formés avec une énergie cinétique considérable et nous pensons que c'est la cause de leur nonobservation par les différents auteurs qui ont étudié la formation des ions négatifs dans $\mathrm{HCl}$. Les énergies cinétiques mesurées sont en accord avec les valeurs calculées à partir de l'expression :

$E_{\mathrm{c}_{\mathrm{th}}}\left(H^{-}\right)=\left(1-\frac{m_{\mathrm{H}}}{m_{\mathrm{HCl}}}\right)\left[V_{\mathrm{e}}-D(\mathrm{H}-\mathrm{Cl})-A \cdot E(\mathrm{H})\right]$

où $V_{\mathrm{e}}$ est l'énergie des électrons incidents,

$D(\mathrm{H}-\mathrm{Cl})$, l'énergie de dissociation de $\mathrm{H}-\mathrm{Cl}(4,43 \mathrm{eV})$ [20],

A. $E(\mathrm{H})$, l'affinité électronique de $\mathrm{H}(-0,754 \mathrm{eV})$ [21].

En effet ce calcul donne :

$$
\begin{array}{lll}
\text { à } & V_{\mathrm{e}}=6,9 \mathrm{eV} & E_{\mathrm{c}_{\mathrm{th}}}\left(\mathrm{H}^{-}\right)=3,15 \mathrm{eV} \\
\text { à } & V_{\mathrm{e}}=9,2 \mathrm{eV} & E_{\mathrm{c}_{\mathrm{th}}}\left(\mathrm{H}^{-}\right)=5,45 \mathrm{eV} .
\end{array}
$$

Ceci indique que les ions $\mathrm{H}^{-}$et les fragments neutres $\mathrm{Cl}$, dans le domaine d'énergie des 2 pics $\mathrm{H}^{-}$, sont formés dans leur état fondamental ${ }^{1} \mathrm{~S}$ et ${ }^{2} \mathrm{P}$ respectivement. Les états de l'ion $\mathrm{HCl}^{-}$, intervenant dans ces processus d'attachement, sont donc les états ${ }^{2} \Sigma$ et ${ }^{2} \Pi$ prévus par les règles de corrélation de Wigner et Witmer.

4. Conclusion et discussion. - L'étude de l'attachement dissociatif sur $\mathrm{HCl}$ et $\mathrm{DCl}$ nous a permis de mettre en évidence trois états de l'ion négatif $\mathrm{HCl}^{-}$ $\left(\mathrm{DCl}^{-}\right)$et en particulier ceux corrélés aux fragments $\mathrm{H}^{-}\left(\mathrm{D}^{-}\right)+\mathrm{Cl}$ qui n'avaient pas encore été observés. Les effets isotopiques ont été mesurés pour les différents processus et nous contestons la valeur de 1,4 obtenue par R. N. Compton et ses collaborateurs pour le rapport

$$
\sigma_{(\mathrm{Cl}-/ \mathrm{HCl})} / \sigma_{(\mathrm{Cl}-/ \mathrm{DCl})} \cdot
$$

Nos expériences indiquent que ce rapport est compris entre 3,9 et 6,1 . 
Dans la «swarm-beam method », l'échantillon à étudier est dilué dans un gaz «porteur» et il est fondamental de connaître la distribution énergétique des électrons dans ce gaz puisque cette grandeur intervient dans le calcul des sections efficaces d'attachement. Ces dernières, de même que les effets isotopiques mesurés par cette méthode, doivent être indépendants du gaz porteur. Cependant, Compton et ses collaborateurs [22] mesurent un effet isotopique de
2,7 pour le processus donnant les ions $\mathrm{Cl}^{-}$lorsque l'argon est utilisé comme gaz porteur. Par ailleurs ils signalent les difficultés dues à l'adsorption de $\mathrm{HCl}$ ou $\mathrm{DCl}$ sur les parois de l'enceinte : peut-être cette adsorption s'accompagne-t-elle d'un échange isotopique. Nous pensons donc que, soit l'incertitude sur la distribution énergétique des électrons, soit l'incertitude sur la composition isotopique, sont à l'origine du désaccord entre les deux mesures expérimentales.

\section{Bibliographie}

[1] Barton, M. A., Phys. Rev. 30 (1927) 614.

[2] Nier, A. O. et Hanson, E. E., Phys. Rev. 50 (1936) 722.

[3] Reese, R. M., Dibeler, V. H., Mohler, F. L., J. Res. Nat. Bur. Stand. 57 (1956) 367.

[4] Gutbier, von H., Neuert, H., Z. Naturforsch. 9a (1954) 335.

[5] Fox, R. E., J. Chem. Phys. 26 (1957) 1281.

[6] Frost, D. C., McDowell, C. A., J. Chem. Phys. 29 (1958) 503.

[7] Buchel'Nixova, I. S., Sov. Phys. JETP 35 (1959) 783.

[8] Compton, R. N., Christophorou, L. G., Dickson, H. W., J. Chem. Phys. 48 (1968) 1949.

[9] Fiquet-Fayard, F., Ziesel, J. P., Azria, R., Tronc, M. et Chitari, J., J. Chem. Phys. 56 (1972) 2540.

[10] Azria, R. et Fiquet-Fayard, F., J. Physique 33 (1972) 663.

[11] Fox, R. F., Hickam, W. M., Grove, D. J., Kueldaas, T., Rev. Sci. Instrum. 26 (1955) 1101.

[12] Ziesel, J. P. et Schulz, G. J. (communication privée).
[13] Stamatovic, A. et Schulz, G. J., J. Chem. Phys. 53 (1970) 2663.

[14] Fiquet-Fayard, F. J. Phys. B (à paraître)

[15] Compton, K. T., Van Voorhis, C. C., Phys. Rev. 26 (1925) 436.

[16] Otvos, J. W., Stevenson, D. P., J. Am. Chem. Soc. 78 (1956) 546.

[17] Lampe, F. W., Franklin, J. L. et Field, F. M., J. Am. Chem. Soc. 79 (1957) 6129.

[18] Rapp, D. et Englander-Golden, P., J. Chem. Phys. 43 (1965) 1464.

[19] Tronc, M., Goursaud, S., Azria, R., Fiquet-Fayard, F., J. Physique 34 (1973) 381.

[20] Herzberg, G., Spectra of Diatomic Molecules (Van Nostrand Cie) 1950.

[21] Page, F. M., Goode, G. C., Negative ions and the Magnetron (Wiley) 1969.

[22] Dickson, H. W., Christophorou, L. G., Compton, R. N., Rapport ORNL-TM. 1724. 